\title{
Influência da abertura de trilhas antrópicas e clareiras naturais na fenologia reprodutiva de Gymnanthes concolor (Spreng.) Müll. Arg. (Euphorbiaceae) ${ }^{1}$
}

\author{
LUIS FERNANDO ALBERTI ${ }^{2,3}$ e L. PATRÍCIA C. MORELLATO ${ }^{2}$
}

(recebido: 20 de dezembro de 2006; aceito: 06 de dezembro de 2007)

\begin{abstract}
Influence of natural gaps and anthropic trails in the reproductive phenology of Gymnanthes concolor (Spreng.) Müll. Arg. (Euphorbiaceae)). In the interior of a seasonal semideciduous forest at Santa Maria, RS, Brazil, we investigated whether a wide and lighter trail differed from a narrow and darker path (darker trail) concerning the reproductive phenology of $G$. concolor. We also examined whether the creation of natural gaps in 2002 affected the incident light and phenology of G. concolor in 2003, answering the questions: (a) Do the trails differ regarding the incident light on the tree canopy and the number of male inflorescences, female flowers, fruits and fruit size (phenological variables)? (b) Is there a relationship between incident light on the tree canopy and the phenological variables? (c) In each trail, do individuals adjacent to gaps differ from individuals far away from gaps regarding the interannual variation in the incident light and phenological variables? In 2002, the incident light was significantly higher on lighter trail than darker trail trees, but trails did not differ in respect to phenological variables. In 2003, incident light and phenological variables did not differ significantly between trees in lighter trail and darker trail. Therefore, the light incidence on lighter trail did not promote a significantly phenological change. The result is supported by the low number of significant regressions between light incidence and phenological variables. Trees adjacent to gaps presented a light incidence variation significantly higher than those trees far away from gaps, but did not differ in respect to interannual variation in phenological variables. Hence, the higher light incidence after the creation of gaps did not affect significantly the phenology of G. concolor as well, which may need even higher amounts of light to respond to gaps or open trails. Our results may give some insight to the design of ecological trails or other open spaces on natural reserves, to reduce its impact on flowering and fruiting production of the species nearby.
\end{abstract}

Key words - anthropic trail, gap creation, Gymnanthes concolor, phenology

RESUMO - (Influência da abertura de trilhas antrópicas e clareiras naturais na fenologia reprodutiva de Gymnanthes concolor (Spreng.) Müll. Arg. (Euphorbiaceae)). Investigamos se no interior de uma floresta estacional semidecidual em Santa Maria, RS, uma trilha clara diferiu de um caminho escuro (trilha escura) quanto à luz incidente e à fenologia reprodutiva de G. concolor e se a abertura de clareiras em 2002 afetou a luz incidente e a fenologia dessa espécie em 2003. Procuramos responder: (a) As trilhas clara e escura diferem quanto à luz incidente na copa das árvores e ao número de inflorescências masculinas, flores femininas, frutos e tamanho de frutos (variáveis fenológicas)? (b) Houve relação entre a luz incidente na copa das árvores e as variáveis fenológicas? (c) Em cada trilha, indivíduos vizinhos e não vizinhos às clareiras diferem quanto à variação interanual da luz incidente e das variáveis fenológicas devido à abertura de clareiras? Em 2002, a luz incidente foi significativamente maior sobre as copas dos indivíduos da trilha clara, mas as trilhas não diferiram significativamente quanto às variáveis fenológicas. Em 2003, os indivíduos na trilha clara não diferiram significativamente daqueles na trilha escura quanto à luz incidente e às variáveis fenológicas. Portanto, a luz incidente na trilha clara não promoveu uma mudança fenológica significativa, o que foi corroborado pelo baixo número de regressões significativas entre luz incidente e as variáveis fenológicas. Os indivíduos vizinhos tiveram taxa de variação da luz incidente significativamente maior do que os não vizinhos, mas não diferiram significativamente quanto às taxas de variação das variáveis fenológicas. Portanto, a maior luz incidente após a abertura de clareiras também não afetou significativamente a fenologia de G. concolor, que dependeria de maiores intensidades de luz para responder à abertura de trilhas ou clareiras. Estes resultados podem nortear as dimensões de futuras trilhas ecológicas ou espaços abertos em reservas naturais, de forma a causarem menor impacto na produção de flores, frutos e sementes em espécies vegetais nas suas proximidades.

Palavras-chave - abertura de clareiras, fenologia, Gymnanthes concolor, trilha antrópica

\section{Introdução}

Os trabalhos sobre clareiras e efeito da fragmentação e de borda na fenologia (e.g. Restrepo \& Vargas 1999)

1. Parte da tese de doutorado do primeiro autor, Programa de Pósgraduação em Biologia Vegetal da Universidade Estadual Paulista (UNESP) de Rio Claro.

2. Universidade Estadual Paulista (UNESP), Instituto de Biociências, Departamento de Botânica, Grupo de Fenologia e Dispersão de Sementes. Av. 24-A, 1515, Bela Vista, 13506-900 Rio Claro, SP, Brasil.

3. atribuem à luz o status de variável chave no entendimento da ecologia de florestas nativas e fragmentadas (Murcia 1995). Contudo, a maioria dos trabalhos sobre a luz em florestas trata da descrição de hábitats (e.g. Bazzaz \& Pickett 1980 e referências neste, Chazdon \& Fetcher 1984, Zaia \& Takaki 1999), não testando sua influência no processo reprodutivo de plantas ou animais (Saunders et al. 1991, Murcia 1995). Conseqüentemente, a resposta fenológica das plantas na natureza decorrente da variação luminosa é pouco conhecida (Ghazoul \& McLeish 2001) 
e ambígua. Johns (1988), Kato \& Hiura (1999), Restrepo et al. (1999) e F.F.D. Neves \& L.P.C. Morellato (dados não publicados) apontaram para o favorecimento da atividade fenológica em condições de maior luminosidade, enquanto Rocha \& Aguilar (2001) relataram o oposto, delegando a causa principal à limitação de polinizadores. Niesenbaum (1993) verificou que Lindera benzoin (L.) Blume (Lauraceae) não teve aumento significativo na produção de frutos, mesmo após o fornecimento de pólen suplementar e apontou a limitação da luz e de nutrientes no solo como fatores importantes para regular a atividade fenológica da referida espécie.

Além da influência ou não dos polinizadores, a incerteza da existência de favorecimento da atividade fenológica em condições de maior luminosidade se deve à complexidade biótica e estrutural das florestas tropicais (Ramos \& Santos 2005), que dificulta a separação da sua influência na fenologia. Por exemplo, as alterações na luz disponível para as plantas devido à abertura de clareiras e ou trilhas modificam a fenologia das espécies vegetais (F.F.D. Neves \& L.P.C. Morellato, dados não publicados, Ramos \& Santos 2005), alterando o número de sementes produzidas e, conseqüentemente, a futura composição florística e densidade de indivíduos das diferentes espécies que ocorrem na floresta (Johns 1988). Compreender até que ponto as variáveis fenológicas medidas em indivíduos vizinhos às clareiras naturais diferem daquelas medidas em trilhas antrópicas possibilita comparar a intensidade do impacto da atividade humana em relação à variação natural na floresta (ver Dahlem $\&$ Boerner 1987).

Dentro deste contexto investigamos, no interior de uma floresta estacional semidecidual, se uma trilha larga e clara (trilha clara) e um caminho estreito e escuro (trilha escura) diferem quanto à luz incidente e a fenologia de Gymnanthes concolor (Spreng.) Müll. Arg. (Euphorbiaceae). Durante o período de estudo, um forte temporal atingiu ambas as trilhas e surgiu a oportunidade de compararmos, a influência da abertura de clareiras na luz e fenologia dos indivíduos de Gymnanthes concolor. Reduzimos a influência de polinizadores e dispersores de sementes no número de frutos observados, por meio da escolha de uma espécie autocórica que pertence a uma família em que a produção de frutos por apomixia é comum (Nigren 1954, Salomão \& Allem 2001). Procuramos responder às perguntas: (a) as trilhas clara e escura diferem quanto à luz incidente na copa das árvores e à fenologia de Gymnanthes concolor? Esperamos que os indivíduos localizados na trilha clara recebam maior quantidade de luz incidente sobre suas copas e apresentem valores mais elevados nas variáveis fenológicas em comparação com aqueles da trilha escura, similar ao observado em borda antrópica por Ramos \& Santos (2005); (b) para cada ano e trilha, existe relação entre a luz incidente na copa e as variáveis fenológicas? Uma vez que a fenologia das árvores responde positivamente a níveis crescentes de luminosidade (Niesenbaum 1993), esperamos que exista relação positiva significativa entre a luz incidente e os valores das variáveis fenológicas; (c) dentro de cada trilha, os indivíduos vizinhos às clareiras diferem dos indivíduos não vizinhos às clareiras quanto à variação da luz incidente e variáveis fenológicas? Devido à marcante diferença na luz entre o interior da floresta e clareiras (Levey 1988, Niesenbaum 1993), esperamos que os indivíduos vizinhos às clareiras apresentem taxa de variação na luz incidente e nas variáveis fenológicas significativamente maiores daquelas registradas nos indivíduos não vizinhos.

\section{Material e métodos}

Espécie estudada - Gymnanthes concolor (Euphorbiaceae) é uma arvoreta característica da floresta estacional semidecidual, ocorre desde o Ceará até o Rio Grande do Sul, atinge de 2 a $10 \mathrm{~m}$ de altura e 18 a $35 \mathrm{~cm}$ de circunferência a altura do peito (CAP), sendo comum no subdossel (Smith et al. 1988), mas pouco freqüente em clareiras (Carvalho et al. 2000) e bordas (Amador \& Viana 2000). Apresenta racemos com 2 a $3 \mathrm{~cm}$ de comprimento, contendo três a seis flores masculinas de cor amarela com até $4 \mathrm{~mm}$ de diâmetro e, comumente, duas flores femininas na base. Os frutos, cápsulas globosas de 5 a $8 \mathrm{~mm}$ de diâmetro (Smith et al. 1988), são secos, deiscentes e autocóricos (Morellato \& Leitão-Filho 1992). Os indivíduos avaliados apresentam baixa variação na CAP, fator que minimiza erros associados à expressão fenológica devido ao vigor das plantas, facilitando desta forma, o isolamento da variável luz, em avaliação neste trabalho.

Área de estudo - O presente estudo foi desenvolvido no Morro do Cerrito, localizado no Município de Santa Maria (2943' S e 5347' W), na região central do Estado do Rio Grande do Sul, Brasil. A região possui clima subtropical do tipo Cfa segundo a classificação climática de Köppen (1948). A temperatura média anual é $23{ }^{\circ} \mathrm{C}$ e a precipitação média anual é de $1823 \mathrm{~mm}$ (estação meteorológica da Universidade Federal de Santa Maria, período de 1980-2003). Na área de estudo, a vegetação é classificada como floresta estacional semidecidual (Veloso et al. 1991) e predominam neossolos litólicos de matriz basáltica (Streck et al. 2002).

As duas trilhas estudadas estão localizadas no interior da floresta do Morro do Cerrito, com 246 m de altitude, exposição predominante norte e 20 ha de sua área recoberta por floresta estacional semidecidual. A trilha larga e clara (trilha clara) possui $700 \mathrm{~m}$ de comprimento e 1 a $5 \mathrm{~m}$ de largura. Foi originada em 1948, a partir de uma estrada utilizada na extração madeireira (comunicação pessoal de Irmão Aloísio Muller). O caminho estreito e escuro (trilha escura) possui 
$500 \mathrm{~m}$ de comprimento e 1 a $2 \mathrm{~m}$ de largura. A trilha clara possui muitas árvores cujas copas não se tocam, criando maior abertura de dossel sobre os indivíduos de Gymnanthes concolor quando comparada com a trilha escura.

As duas trilhas foram caracterizadas quanto à luminosidade durante caminhada com um luxímetro LI-210 Photometric Sensor constituído de um sensor pontual posicionado sobre a palma da mão a 1,30 $\mathrm{m}$ de altura, parando a aproximadamente cada $15 \mathrm{~s}$ para fazer a leitura. O sensor coletou 209 amostras na trilha clara e 244 amostras na trilha escura, alternando $30 \mathrm{~min}$. em cada trilha, entre as $13 \mathrm{~h} 30 \mathrm{~min}$ e $15 \mathrm{~h} 30 \mathrm{~min}$ do dia $1^{\circ}$ de outubro de 2002 . A luz incidente foi significativamente maior na trilha clara $($ mediana $=0,7 \mathrm{Klx}$ ) do que na trilha escura (mediana $=0,4 \mathrm{Klx}$, Mann-Whitney $U=9, P<0,001)$, comprovando a existência de dois ambientes distintos. A trilha clara apresentou maior variação de luz incidente $(25-75$ percentis $=0,4-3,0)$ em relação à trilha escura (25-75 percentis $=0,3-0,8 \mathrm{Klx})$.

Amostragem dos indivíduos - Em ambas as trilhas foram amostrados todos os indivíduos de Gymnanthes concolor com circunferência a altura do peito (CAP) maior que $31,4 \mathrm{~cm}$ e que se encontravam a uma distância menor que $10 \mathrm{~m}$ em relação ao centro das mesmas. A amostragem de indivíduos acima de 31,4 cm de CAP reduz a freqüência de valores zero nas variáveis reprodutivas, aumentando a confiabilidade dos resultados das análises estatísticas. A amostragem totalizou $500 \times 10 \mathrm{~m}(0,5 \mathrm{ha})$ em cada trilha, sendo que foram encontrados 17 indivíduos na trilha clara e 15 na trilha escura. Dentre os indivíduos amostrados, foram selecionados oito em cada trilha, de acordo com a facilidade de acesso à copa. Para cada indivíduo selecionado foram avaliados, nos anos de 2002 e 2003, uma vez na floração e outra na frutificação, na porção mediana da copa, um ramo de $1,0 \times 0,5 \times 0,5 \mathrm{~m}$ em cada ponto cardeal. Em cada ramo (no caso das variáveis $\mathrm{Y}_{1}$ a $\mathrm{Y}_{3}$ e $\mathrm{X}_{2}$ ) e indivíduo $\left(\mathrm{X}_{1}\right)$ foram quantificadas as seguintes variáveis dependentes ou fenológicas $(\mathrm{Y})$ e independentes $(\mathrm{X})$ :

$\mathrm{Y}_{1}$ - Número de inflorescências masculinas; $\mathrm{Y}_{2}-$ número de flores femininas; $\mathrm{Y}_{3}-$ número de frutos, $\mathrm{Y}_{4}-$ tamanho de frutos.

Para cada indivíduo selecionado, tomamos seu tronco como referência central para a instalação de uma unidade amostral de 5 x $5 \mathrm{~m}$ no chão, subdividida em quatro subunidades de 2,5 x 2,5 m. Em cada subunidade, coletamos todos os frutos em bom estado encontrados durante $5 \mathrm{~min}$. de busca. Medimos o comprimento e largura de um a cinco frutos, conforme a disponibilidade dos mesmos, somente em 2002; $X_{1}$ - circunferência à altura do peito (CAP) - medida em todos os indivíduos amostrados; $\mathrm{X}_{2}$ - luz incidente na copa da árvore, amostrada a $4 \mathrm{~m}$ de altura com o sensor do luxímetro LI-210 posicionado sobre uma pequena plataforma acoplada à ponta de uma vara, por meio de quatro tomadas por ramo, cuja posição em cada ramo definimos de acordo com a facilidade de manuseio do sensor. Alternamos a coleta de dados em indivíduos na trilha clara e escura, entre as 11h30min e 15h30min em um único dia de agosto de 2002.
Para as variáveis $\mathrm{Y}_{1}$ a $\mathrm{Y}_{3}, \mathrm{X}_{1}$ e $\mathrm{X}_{2}$, usamos a somatória das medidas dos quatro ramos e calculamos a mediana entre os indivíduos por ambiente nas tabelas e teste $U$ de MannWhitney (Zar 1996); para a variável $Y_{4}$, em cada indivíduo, calculamos a média das quatro subunidades, sendo que obtivemos o valor de cada subunidade por meio da média do tamanho dos frutos encontrados na mesma. Posteriormente calculamos a mediana entre os indivíduos.

Em 2002 e 2003, comparamos as duas trilhas quanto à soma de postos dos valores da luz incidente e às variáveis fenológicas medidas nas copas dos indivíduos de Gymnanthes concolor por meio do teste $U$ de Mann-Whitney, conforme Zar (1996). Em ambas as trilhas e anos testamos a relação entre as fenofases reprodutivas e luz por meio de regressão linear simples, considerando $\mathrm{Y}_{1}$ a $\mathrm{Y}_{3}$ como variáveis dependentes e $X_{2}$ como independente (Zar 1996).

Para testar a influência da abertura de clareiras ocorrida em 11 de setembro de 2002, identificamos entre os indivíduos selecionados em cada trilha aqueles vizinhos e não vizinhos às clareiras e calculamos a taxa de variação dos valores, ou seja, os valores obtidos após a abertura de clareiras (2003) menos os valores obtidos antes da abertura das clareiras (2002) das variáveis $\mathrm{Y}_{1}$ a $\mathrm{Y}_{3}$ e $\mathrm{X}_{2}$ para cada um dos quatro indivíduos vizinhos e não vizinhos às clareiras da trilha clara e três indivíduos vizinhos às clareiras e cinco não vizinhos na trilha escura. Consideramos indivíduos vizinhos às clareiras aqueles cuja base do seu tronco delimitava a abertura da clareira (Runkle 1981).

Para cada trilha comparamos indivíduos vizinhos e não vizinhos às clareiras quanto à taxa de variação da luz incidente e das variáveis reprodutivas por meio do teste $U$ de MannWhitney. A existência de diferença significativa no teste $U$ indicaria que, dentro de uma dada trilha, a soma dos postos das taxas de variação das variáveis $\mathrm{Y}_{1}$ a $\mathrm{Y}_{3}$ e $\mathrm{X}_{2}$ diferia significativamente entre os indivíduos vizinhos e não vizinhos às clareiras.

\section{Resultados}

Gymnanthes concolor floresceu de agosto a setembro e frutificou de agosto a novembro e a maioria dos frutos foi produzida por apomixia (L.F. Alberti, dados não publicados) e não apresentou diferença significativa entre a trilha clara e a trilha escura quanto ao posto médio dos valores de CAP (tabela 1).

Em 2002, a luz incidente na copa foi significativamente maior nos indivíduos localizados na trilha clara em relação aqueles na trilha escura (tabela 1). Não houve diferenças significativas entre as trilhas quanto ao número de inflorescências masculinas, flores femininas e frutos produzidos (tabela 1). No entanto, em 2002 e 2003 houve uma produção maior de flores masculinas na trilha clara. Os indivíduos localizados na trilha clara não diferiram significativamente daqueles da trilha escura quanto ao 
Tabela 1. Luz incidente, variáveis fenológicas e circunferência à altura do peito (CAP) de Gymnanthes concolor nas trilhas clara e escura (mediana e entre parênteses os 25 e 75 percentis), nos dois anos de estudo, em floresta estacional semidecidual, em Santa Maria, RS. ( $-=$ Valores não estimados).

Table 1. Incident light, phenological variables and circumference at breast high (CAP) of Gymnanthes concolor in the open and dark trails (median and 25 and 75 percentiles inside parenthesis), in the two years of study, in the seasonal semideciduous forest in Santa Maria, RS. (- = Not estimated).

\begin{tabular}{|c|c|c|c|c|}
\hline & \multicolumn{2}{|c|}{ Trilha clara } & \multicolumn{2}{|c|}{ Trilha escura } \\
\hline & 2002 & 2003 & 2002 & 2003 \\
\hline Luz incidente na copa\# (Klx) & $\begin{array}{c}60,3 \\
(37,5-91,4)^{*}\end{array}$ & $\begin{array}{c}67,8 \\
(28,1-211,7)\end{array}$ & $\begin{array}{c}13,2 \\
(10,3-20,2)\end{array}$ & $\begin{array}{c}41,81 \\
(28,2-50,6)\end{array}$ \\
\hline Número de inflorescências masculinas & $\begin{array}{c}40,0 \\
(31,5-48,5)\end{array}$ & $\begin{array}{c}81,5 \\
(67,5-99,5)\end{array}$ & $\begin{array}{c}30,0 \\
(16,0-35,5)\end{array}$ & $\begin{array}{c}65,5 \\
(49,0-95,5)\end{array}$ \\
\hline Número de flores femininas ${ }^{\#}$ & $\begin{array}{c}7,0 \\
(3,0-14,0)\end{array}$ & $\begin{array}{c}22,5 \\
(11,5-26,5)\end{array}$ & $\begin{array}{c}6,0 \\
(3,5-7,5)\end{array}$ & $\begin{array}{c}14,0 \\
(7,0-20,0)\end{array}$ \\
\hline Número de frutos $\#$ & $\begin{array}{c}4,0 \\
(3,5-5,0)\end{array}$ & $\begin{array}{c}18,5 \\
(10,5-23,0)\end{array}$ & $\begin{array}{c}5,5 \\
(4,0-8,0)\end{array}$ & $\begin{array}{c}15,5 \\
(10,5-19,5)\end{array}$ \\
\hline Tamanho de frutos ${ }^{\#}$ & $\begin{array}{c}0,8 \\
(0,7-0,9)\end{array}$ & - & $\begin{array}{c}0,7 \\
(0,6-0,8)\end{array}$ & - \\
\hline $\mathrm{CAP}^{\dagger}$ & $\begin{array}{c}36,0 \\
(32,0-39,0)\end{array}$ & - & $\begin{array}{c}35,0 \\
(31,5-36,0)\end{array}$ & - \\
\hline
\end{tabular}

† Número de indivíduos $=17$ na trilha clara e 15 na trilha escura .

\# Número de indivíduos $=8$ na trilha clara e 8 na trilha escura.

* $P<0,01=$ Valor significativo segundo o teste $U$ de Mann-Whitney.

tamanho dos frutos (tabela 1). Em 2003, os indivíduos localizados na trilha clara não diferiram significativamente daqueles localizados na trilha escura quanto à luz incidente ou qualquer variável fenológica estudada.

Ao contrário do esperado, os modelos de regressão linear simples testados não mostraram relação significativa entre a luz e variáveis reprodutivas (figura 1). Somente o número de flores femininas produzidas pelos indivíduos na trilha escura em 2002 apresentou relação negativa significativa com a luz incidente (figura 1B).

Na comparação entre indivíduos vizinhos e não vizinhos às clareiras quanto à taxa de variação interanual na luz incidente e das variáveis fenológicas observamos que, tanto na trilha clara quanto na trilha escura, os indivíduos vizinhos às clareiras tiveram taxa de variação de luz incidente significativamente maior do que os indivíduos não vizinhos, mas não diferiram significativamente quanto às taxas de variação das variáveis fenológicas (tabela 2).

\section{Discussão}

Ao contrário do previsto, a trilha clara não diferiu significativamente da trilha escura quanto às variáveis fenológicas em nenhum dos anos de estudo. A maior luz

\footnotetext{
$\dagger$ Number of individuals $=17$ in the open trail and 15 in the dark trail. \# Number of individuals $=8$ in the open trail and 8 in the dark trail. * $P<0,01=$ Significative value according to the Mann-Whitney $U$ test.
}

incidente sobre a copa de Gymnanthes concolor na trilha clara, aparentemente, não favoreceu a atividade reprodutiva quando comparada com a trilha escura. Valores superiores de luz na borda (Zaia \& Takaki 1999, Hernandes et al. 2004) favorecem a maior produtividade de flores e frutos em árvores nessas condições (F.F.D. Neves \& L.P.C. Morellato, dados não publicados, Quesada et al. 2004) quando comparadas com aquelas árvores localizadas dentro da floresta (Carvalho et al. 2000). Árvores isoladas possuem atividade reprodutiva ainda maior do que na borda, sugerindo que uma combinação de quantidades excepcionais de luz incidente e de espaço físico para suas raízes favoreceria a obtenção de nutrientes e conseqüentemente a atividade reprodutiva nessas condições (Fuchs et al. 2001). Portanto, aparentemente os indivíduos de Gymnanthes concolor da trilha clara necessitariam de maior quantidade de luz para que sua atividade fenológica fosse significativamente maior do que aquela observada nos indivíduos da trilha escura. A observação, na área estudada, de alguns indivíduos isolados com abundante produção de flores reforça esta idéia.

A tendência de maior produção de inflorescências masculinas na trilha clara em ambos os anos sugere que níveis de luz incidente superiores àqueles observados 

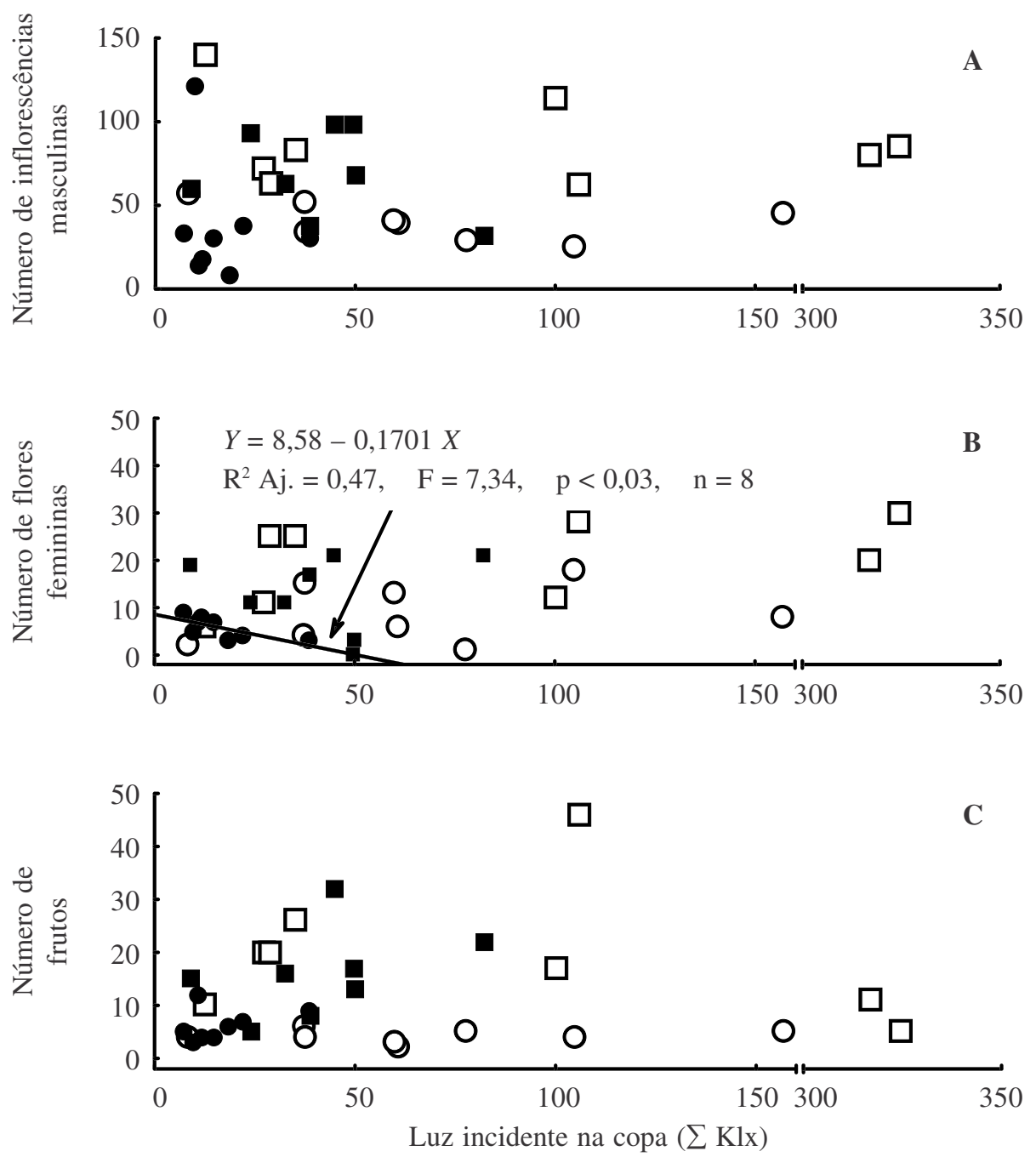

Figura 1. Relação entre a variação na luz incidente na copa e o número de inflorescências masculinas (A), flores femininas (B) e frutos (C) em indivíduos de Gymnanthes concolor em floresta estacional semidecidual em Santa Maria, RS. Número de indivíduos $=8$, para cada trilha e ano (círculos $=2002$; quadrados $=2003)$. Símbolos: $O=$ trilha clara; $\bullet=$ trilha escura; $\square=$ trilha clara, $\mathbf{\square}=$ trilha escura.

Figure 1. Relationship between incident light on the tree crown and the number of (A) male inflorescences, (B) female flowers and (C) fruits on trees of Gymnanthes concolor at the seasonal semideciduous forest in Santa Maria, RS. Number of trees $=8$ in each trail and year (circle $=2002$; square $=2003$. Symbols: $O=$ open trail; $\bullet=$ dark trail; $\square=$ open trail, $\mathbf{\square}=$ dark trail .

no presente estudo poderiam favorecer a produção de estruturas que são menos custosas para a planta, como proposto por Fuchs et al. (2001), Quesada et al. (2004) e Holland et al. (2004). Como consequiência, poderíamos ter um favorecimento da reprodução cruzada e trocas genéticas efetuadas pelos grãos de pólen, especialmente se considerarmos que em Gymnanthes concolor grande parte dos frutos é formada por apomixia (L.F. Alberti, dados não publicados).

Ao contrário do esperado, a maioria dos modelos lineares simples testados para a relação entre a luz incidente e as variáveis fenológicas não foram significativos, sugerindo que a grande heterogeneidade da luz incidente no nível de micro-habitat em florestas tropicais torna difícil isolar e modelar sua influência na resposta reprodutiva de espécies vegetais (Kato \& Hiura 1999, Ramos \& Santos 2005). Ainda, sugere que a fenologia de Gymnanthes concolor é influenciada também por fatores diferentes da luz incidente. Neste sentido, Pinero et al. (1982) sugerem que a frutificação de Astrocaryum mexicanum L. (Arecaceae) estaria também relacionada à disponibilidade de nutrientes no solo.

A abertura de clareiras aumenta a luz incidente (Chazdon \& Fetcher 1984) e favorece a produção de frutos (Blake \& Hoppes 1986, Dahlem \& Boerner 1987, 
Tabela 2. Variação interanual das variáveis fenológicas devida à abertura de clareiras, em indivíduos de Gymnanthes concolor vizinhos (VC) e não vizinhos (NV) às clareiras (mediana e entre parênteses os 25 e 75 percentis), em floresta estacional semidecídual, Santa Maria, RS.

Table 2. Interannual variation of the phenological variables due to the gap creation, observed on Gymnanthes concolor trees adjacent (VC) and not adjacent (NV) to gaps (median and 25 and 75 percentiles inside parenthesis), in the seasonal semideciduous forest at Santa Maria, RS.

\begin{tabular}{|c|c|c|c|c|}
\hline & \multicolumn{2}{|c|}{ Trilha clara } & \multicolumn{2}{|c|}{ Trilha escura } \\
\hline & $\begin{array}{c}\mathrm{VC} \\
(n=4)\end{array}$ & $\begin{array}{c}\mathrm{NV} \\
(n=4)\end{array}$ & $\begin{array}{c}\mathrm{VC} \\
(n=3)\end{array}$ & $\begin{array}{c}\mathrm{NV} \\
(n=5)\end{array}$ \\
\hline Luz incidente na copa (Klx) & $\begin{array}{c}118,0 * \\
(36,5 ; 212,0)\end{array}$ & $\begin{array}{c}-37,5 \\
(-63,2 ; 19,2)\end{array}$ & $\begin{array}{c}40,7 * \\
(34,9 ; 43,8)\end{array}$ & $\begin{array}{c}22,8 \\
(14,2 ; 25,3)\end{array}$ \\
\hline Número de inflorescências masculinas & $\begin{array}{c}40,5 \\
(34,0 ; 62,0)\end{array}$ & $\begin{array}{c}42,5 \\
(40,0 ; 52,5)\end{array}$ & $\begin{array}{c}54,0 \\
(2,0 ; 68,0)\end{array}$ & $\begin{array}{c}30,0 \\
(20,0 ; 52,0)\end{array}$ \\
\hline Número de flores femininas & $\begin{array}{c}13,5 \\
(8,5 ; 18,0)\end{array}$ & $\begin{array}{c}9,0 \\
(7,5 ; 11,0)\end{array}$ & $\begin{array}{c}-4,0 \\
(-7,0 ; 18,0)\end{array}$ & $\begin{array}{c}9,0 \\
(6,0 ; 16,0)\end{array}$ \\
\hline Número de frutos & $\begin{array}{c}7,5 \\
(3,0 ; 25,5)\end{array}$ & $\begin{array}{c}15,5 \\
(13,0 ; 19,5)\end{array}$ & $\begin{array}{c}13,0 \\
(1,0 ; 13,0)\end{array}$ & $\begin{array}{c}9,0 \\
(4,0 ; 11,0)\end{array}$ \\
\hline
\end{tabular}

Levey 1988). No presente estudo, a abertura de clareiras, embora tenha proporcionado aumento significativo na luz incidente na copa dos indivíduos vizinhos às clareiras nas duas trilhas, não promoveu aumento significativo na atividade fenológica nos indivíduos de Gymnanthes concolor vizinhos às clareiras em relação aos não vizinhos às clareiras. As clareiras abertas possuem, em sua grande maioria, menos que $80 \mathrm{~m}^{2}$. Não observamos nenhuma clareira grande (maior que $200 \mathrm{~m}^{2}$ sensu Muniz 2004) próxima aos indivíduos de Gymnanthes concolor amostrados, que propiciaria uma maior quantidade de luz incidente, podendo afetar a fenologia da espécie. Em floresta estacional semidecidual na Estação Ecológica de Caetetus, SP, Muniz (2004) verificou que as clareiras pequenas estão expostas a menor quantidade de luz quando comparadas às clareiras grandes. Estudando várias espécies de arbustos na Costa Rica, Levey (1988) observou número significativamente maior de indivíduos com frutos em clareiras maiores que $320 \mathrm{~m}^{2}$ em relação à clareiras menores que $80 \mathrm{~m}^{2}$. Em uma clareira com $900 \mathrm{~m}^{2}$, distante das trilhas estudadas e aberta também em 11 de setembro de 2002, foram observados indivíduos de Gymnanthes concolor com floração copiosa em abril, fora da época normal de florescimento da espécie. Portanto, para que a atividade fenológica de Gymnanthes concolor seja aumentada significativamente em decorrência da abertura de clareiras, talvez sejam necessárias clareiras de maiores dimensões do que as consideradas neste estudo.

Os resultados do presente estudo sugerem que as respostas das espécies a abertura de trilhas e a incidência de luz podem ser diferentes do previsto pela literatura corrente. São necessários ambientes como trilhas mais largas ou clareiras maiores para que a luz incidente alcance níveis capazes de modificar significativamente a fenologia de Gymnanthes concolor bem como testar experimentalmente até que ponto a sua fenologia (principalmente a formação de flores femininas e frutos) responde ao acréscimo de luz incidente ou é limitada por nutrientes no solo. Adicionalmente, para as condições do presente estudo, a abertura de trilhas com até $5 \mathrm{~m}$ de largura e a abertura de clareiras com menos de $80 \mathrm{~m}^{2}$ não foram capazes de afetar significativamente a fenologia de Gymnanthes concolor. O valor da largura da trilha estudada pode nortear as dimensões de futuras trilhas ecológicas ou espaços abertos em parques, de forma que a abertura dos mesmos cause menor impacto na produção de flores, frutos e sementes em espécies vegetais nas suas proximidades.

Agradecimentos - Os autores agradecem à FAPESP, pela bolsa de doutorado concedida a Luis Fernando Alberti e ao CNPq pela bolsa de produtividade em pesquisa e taxa de bancada associada, concedidos a L. Patrícia C. Morellato. O Laboratório de Fenologia onde este trabalho foi desenvolvido recebe apoio da FAPESP e CNPq.

\section{Referências bibliográficas}

AMADOR, D.B. \& VIANA, V.M. 2000. Dinâmica de capoeiras baixas na restauração de um fragmento florestal. Scientia Forestalis 57:69-85. 
BAZZAZ, F.A. \& PICKETT, S.T.A. 1980. Physiological ecology of tropical succession: a comparative review. Annual Review of Ecology and Systematics 11:287-310.

BLAKE, J.G.H. \& HOPPES, W.G. 1986. Influence of resource abundance on use of tree-fall gaps by birds in an isolated woodlot. The Auk 103:328-340.

CARVALHO, L.M.T., FONTES, M.A.L. \& OLIVEIRA-FILHO, A.T. 2000. Tree species distribution in canopy gaps and mature forest in an area of cloud forest of Ibitipoca range, south-eastern Brazil. Plant Ecology 149:9-22.

CHAZDON, R.L. \& FETCHER, N. 1984. Photosynthetic light environments in a lowland tropical rainforest in Costa Rica. Journal of Ecology 72:553-564.

DAHLEM, T.S. \& BOERNER, R.E.J. 1987. Effects of canopy light gap on the growth and reproduction of Geranium maculatum. Canadian Journal of Botany 65:242-245.

FUCHS, E.J., LOBO, J.A. \& QUESADA, M. 2001. Effects of forest fragmentation and flowering phenology on the reproductive success and mating patterns of the tropical dry forest tree Pachira quinata. Conservation Biology 17:1523-1539.

GHAZOUL, J. \& MCLEISH, M. 2001. Reproductive ecology of tropical forest trees in logged and fragmented habitats in Thailand and Costa Rica. Plant Ecology 153:335345.

HERNANDES, J.L., PEDRO-JUNIOR, M.J. \& BARDIN, L. 2004. Variação estacional da radiação solar em ambiente externo e no interior de floresta semidecídua. Revista Árvore 28:67-172.

HOLLAND, J.N., BRONSTEIN, J.L. \& DEANGELIS, D.L. 2004. Testing hypotheses for excess flower production and low fruit-to-flower ratios in a pollinating seedconsuming mutualism. Oikos 105:633-640.

JOHNS, A.D. 1988. Effects of "selective" timber extraction on rain forest structure and composition and some consequences for frugivores and folivores. Biotropica 20:31-37.

KATO, E. \& HIURA, T. 1999. Fruit set in Styrax obassia (Styracaceae): the effect of light availability, display size, and local floral density. American Journal of Botany 86:495-501.

KÖPPEN, W. 1948. Climatologia: con un estudio de los climas de la tierra. Fundo de Cultura Econômica, México.

LEVEY, D.J. 1988. Spatial and temporal variation in Costa Rican fruit and fruit-eating bird abundance. Ecological Monographs 58:251-269.

MORELLATO, L.P.C. \& LEITÃO-FILHO, H.F. 1992. Padrões de frutificação e dispersão na Serra do Japi. In História natural da Serra do Japi: ecologia e preservação de uma área florestal no Sudeste do Brasil. (L.P.C. Morellato, org.) Editora da Unicamp, Campinas. p.112-140.

MUNIZ, M.R.A. 2004. Estudo do regime de luz nas quatro principais formações fitogeográficas no Estado de São Paulo durante o inverno do ano de 2003. Dissertação de mestrado, Universidade Estadual de Campinas, Campinas.
MURCIA, C. 1995. Edge effects in fragmented forests. Trends in Ecology and Evolution 10:58-62.

NIESENBAUM, R.A. 1993. Light or pollen - seasonal limitations on female reproductive success in the understory shrub Lindera benzoin. Journal of Ecology 81:315-323.

NIGREN, A. 1954. Apomixis in Angiosperms. Part II. Botanical Review 20:577-649.

PINERO, D., SARUKHAN, J. \& ALBERDI, P. 1982. The costs of reproduction in a tropical palm, Astrocaryum mexicanum. Journal of Ecology 70:473-481.

QUESADA, M., STONER, K.E., LOBO, J.A., HERRERÍASDIEGO, Y., PALACIOS-GUEVARA, C., MUNGUÍAROSAS, M.A., O-SALAZAR, K.A. \& ROSASGUERRERO, V. 2004. Effects of forest fragmentation on pollinator activity and consequences for plant reproductive success and mating patterns in bat-pollinated Bombacaceae trees. Biotropica 36:131-138.

RAMOS, F.N. \& SANTOS, F.M. 2005. Phenology of Psychotria tenuinervis (Rubiaceae) in Atlantic Forest fragments. Canadian Journal of Botany 83:1305-1316.

RESTREPO, C. \& VARGAS, A. 1999. Seeds and seedlings of two neotropical montane understory shrubs respond differently to anthropogenic edges and treefall gaps. Oecologia 119:419-426.

RESTREPO, C., GOMEZ, N. \& HEREDIA, S. 1999. Anthropogenic edges, treefall gaps, and fruit-frugivore interactions in a neotropical montane forest. Ecology 80:668-685.

ROCHA, O.J. \& AGUILAR, G. 2001. Reproductive biology of the dry forest tree Enterolobium cyclocarpum (Guanacaste) in Costa Rica: a comparison between trees left in pastures and trees in continuous forest. American Journal of Botany 88:1607-1614.

RUNKLE, J.R. 1981. Gap regeneration in some old-growth forests of the eastern United States. Ecology 62:1041-1051.

SALOMÃO, A.N. \& ALLEM, A.C. 2001. Polyembryony in angiospermous trees of the Brazilian Cerrado and Caatinga vegetation. Acta Botanica Brasilica 15:369-378.

SAUNDERS, D.A., HOBBS, R.J. \& MARGULES, C.R. 1991. Biological consequences of ecosystem fragmentation: a review. Conservation Biology 5:18-32.

SMITH, L.B., DOWNS, R.J. \& REITZ, R. 1988. Euforbiáceas. In Flora ilustrada catarinense. (R. Reitz, ed.). Herbário Barbosa Rodrigues, Itajaí.

STRECK, E.V., KÄMPF, N., DALMOLIN, R.S.D. \& KLANT, E. \& SCHNEIDER, F. 2002. Solos do Rio Grande do Sul. Editora da UFRGS, Porto Alegre.

VELOSO, H.P., RANGEL FILHO, A.L.R. \& ALVES-LIMA, J.C. 1991. Classificação da vegetação brasileira adaptada a um sistema universal. Fundação IBGE, Rio de Janeiro.

ZAIA, J.E. \& TAKAKI, M. 1999. A relação V/VE da radiação solar sob o dossel de uma área de Mata Atlântica. Acta Botanica Brasilica 13:43-48.

ZAR, J.H. 1996. Biostatistical analysis. Prentice-Hall, New Jersey. 